\title{
Interaction of Polyphenolic Metabolites with Human Serum Albumin: A Circular Dichroism Study
}

\author{
Akiko Nozaki, ${ }^{a}$ Toshikiro Kimura, ${ }^{b}$ Hideyuki Ito, ${ }^{a}$ and Tsutomu Hatano*, $a$ \\ a Department of Pharmacognosy, Okayama University Graduate School of Medicine, Dentistry and Pharmaceutical \\ Sciences; and bepartment of Pharmaceutics, Okayama University Graduate School of Medicine, Dentistry and \\ Pharmaceutical Sciences; Tsushima, Okayama 700-8530, Japan.
}

Received May 26, 2009; accepted July 1, 2009; published online July 2, 2009

\begin{abstract}
Binding sites of polyphenolic compounds on human serum albumin (HSA) were investigated using induced Cotton effects on the circular dichroism (CD) spectra. Polyphenolic compounds used in this study are known to be metabolites from tannins and their related polyphenols in food and medicinal plants. The present investigation revealed that the structural differences markedly affected the binding of the compounds to HSA. Protocatechuic acid, together with its methylated compounds vanillic and isovanillic acids, were assigned to be bound to sites I and II of HSA, based on the competitive relationships with site-I-binding phenylbutazone (PB) and site-IIbinding diazepam (DP). 4-O-Methylgallic acid, which is the metabolite from gallic acid, was bound to site I on HSA, while gallic acid did not affect the binding of PB and DP at the concentration examined. Neither ellagic acid nor its metabolite urolithin A was competitive with PB and DP on HSA. The addition of digitoxin did not affect the induced $C D$ of the polyphenolic acids examined.
\end{abstract}

Key words polyphenol; serum albumin; interaction; circular dichroism; gallic acid; protocatechuic acid

Recent investigations have revealed the health benefits of polyphenolic constituents in dietary and medicinal plants. ${ }^{1,2}$ The mechanism of action of the polyphenols corresponding to those effects has been, at least in part, ascribable to polyphenol interaction with biomolecules, especially proteins. Several studies have concentrated on the interaction related to (-)-epigallocatechin gallate (EGCG), the major constituent of tea. ${ }^{3,4}$ We previously reported that EGCG binds to human serum albumin (HSA). ${ }^{5}$

In the previous report, we noted the utility of circular dichroism (CD) spectra to clarify the binding of polyphenols with HSA, including the binding sites on HSA in relation to the competition with drugs having specific binding sites on the protein. EGCG has been shown to bind to sites I and II in HSA. The results suggested the possibility of a drug interaction concerning competition. ${ }^{5}$ )

The metabolic fates of plant polyphenols have been investigated in many studies. Proanthocyanidins and catechins yield phenolic acids such as protocatechuic acid, $\left.{ }^{6}\right)$ and methylation of phenolic acids, attributable to the enzyme catechol-O-methyl transferase (COMT), is also an important metabolic process of those phenolic compounds. Tannic acid, a gallotannin mixture, is metabolized into gallic acid and its further methylation gives 4-O-methylgallic acid (5-hydroxyisovanillic acid) ${ }^{7,8)}$ while metabolism of ellagitannins yields ellagic acid, or further reductive changes produce urolithin A (3,8-dihydroxy-6H-dibenzo[ $b, d]$-pyran-6-one) and related

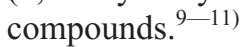

The present study thus treated the binding of those phenolic compounds shown in Fig. 1, which are regarded as metabolites from tannins and related compounds. ${ }^{6-11)}$ The experiments revealed noticeable differences in the binding behavior due to subtle differences in the structures of the compounds as discussed below.

\footnotetext{
Experimental

Reagents HSA (A3782, the fatty acid free grade, $\geq 99 \%$ ), phenylbutazone (PB), digitoxin (DG), ellagic acid, and vanillic acid were purchased from Sigma (St. Louis, MO, U.S.A.). Diazepam (DP) and protocatechuic
}

acid were from Wako (Osaka, Japan), and gallic acid, 4-O-methylgallic acid, and isovanillic acid were, respectively, the products of Ishidzu (Osaka, Japan), Extrasynthèse (Genay, France), and Aldrich (Milwaukee, WI, U.S.A.).

Urolithin A was synthesized via coupling of resorcinol and methyl 2bromo-5-hydroxybenzoate, which was obtained by bromination of $m$-hy-
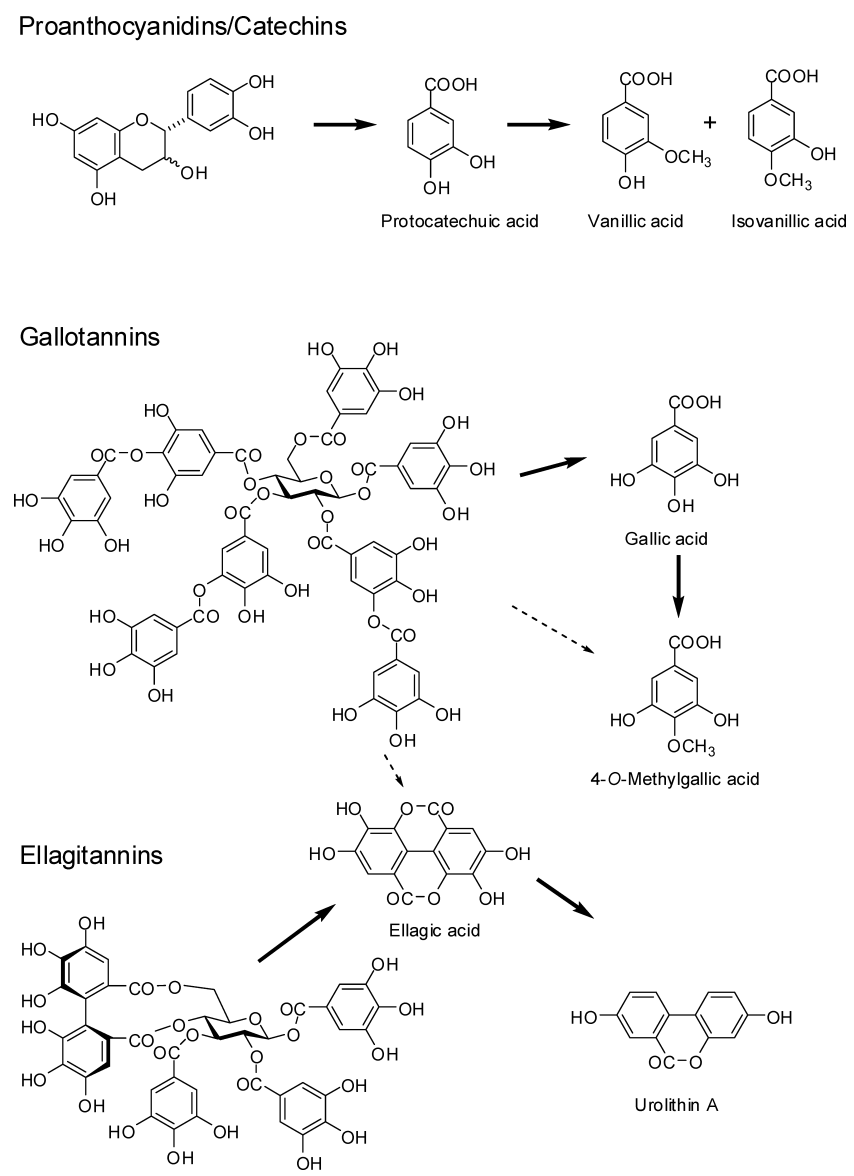

Fig. 1. Metabolic Relationship of the Polyphenolic Compounds and Hydrolyzable Tannins/Flavans 
droxybenzoic acid followed by methylation according to a previous report. ${ }^{12)}$

CD Spectral Measurements CD spectra were recorded on a JASCO J$720 \mathrm{~W}$ spectropolarimeter (Tokyo, Japan) using a $0.1-\mathrm{cm}$ path-length cell at $288-291 \mathrm{~K}$. The time constant, scan speed, resolution, and sensitivity were set at $1 \mathrm{~s}, 100 \mathrm{~nm} / \mathrm{min}, 1.0 \mathrm{~nm}$, and $5 \mathrm{mdeg}$, respectively. Twelve scanned spectra between 355 and $245 \mathrm{~nm}$ were accumulated for spectral measurements. When changes in amplitudes of the induced Cotton effects were estimated, the resolution was set at $0.5 \mathrm{~nm}$ and data from 32 scans were given as the means of the three points around the indicated wavelength.

The wavelength was originally set considering the minimum overlap of the induced CD, when the induced CD for the HSA-drug or HSA-phenolic compound overlapped. Drugs used in this study were those with specific binding sites on HSA to determine the specific binding sites of the phenolic compounds. The induced CD for the HSA-drug or HSA-phenolic compound was expressed by the following equation, since the drugs and the phenolic compounds used in this study, except for DG, do not have their own CD.

$$
\text { induced } \mathrm{CD}=\mathrm{CD}_{\mathrm{HSA}+\text { drug (or phenolic compound) }}-\mathrm{CD}_{\mathrm{HSA}}
$$

All experiments were performed in a solution of $0.1 \mathrm{M} \mathrm{K} \mathrm{KPO}_{4}-\mathrm{KH}_{2} \mathrm{PO}_{4}$ buffer ( $\mathrm{pH} 7.0$ ). The final concentration of HSA was set at $6.02 \times 10^{-5} \mathrm{M}$. The concentrations of drugs with known binding sites, PB (site I) and DP (site II), varied in the range of $0-3.0 \times 10^{-4} \mathrm{M}$, and the effects of adding $0.15 \mathrm{mg} / \mathrm{ml}$ of the phenolic compounds (final concentration) were examined.

Since DG, which is bound to site III on HSA, does not show noticeable induced $\mathrm{CD}$, concentrations of phenolic acids were varied, and the effects of adding $1.96 \times 10^{-4} \mathrm{M}$ of DG were examined.

The competitive/noncompetitive relationships of the drugs and phenolic compounds were analyzed based on Rosen's method ${ }^{13)}$ using double-reciprocal plots. $^{14)}$

The molar concentration of the drug bound to HSA, $D_{\mathrm{b}}$, was estimated from the tangential line of the curve indicating the relationship of the concentration of the drug and the amplitude of the induced CD. The molar concentration of the drug unbound, $D_{\mathrm{f}}$, was estimated by subtraction of $D_{\mathrm{b}}$ from the concentration of the drug added.

The double-reciprocal relationship between $1 / D_{\mathrm{f}}$ and $1 / r$ is represented by the equation

$$
\frac{1}{r}=\frac{1}{n K} \cdot \frac{1}{D_{\mathrm{f}}}+\frac{1}{n}
$$

where $r$ indicates the number of the molecule of the drug bound to a molecule of HSA, corresponding to $D_{\mathrm{b}} / P_{\mathrm{t}} . P_{\mathrm{t}}$ represents the molar concentration of HSA added (bound and unbound, in total). The apparent binding constant $(K)$ for each combination of drug-HSA was calculated from this relationship. ${ }^{5)}$

In the presence of a polyphenolic additive $(A)$, the equation for the double-reciprocal plots is expressed to be

$$
\frac{1}{r}=\frac{1}{n K^{\prime}} \cdot \frac{1}{D_{\mathrm{f}}}+\frac{1}{n}
$$

where $K^{\prime}$ represents the apparent binding constant in the presence of the drug and the polyphenolic additive, and corresponds to

$$
\frac{K}{1+K_{\mathrm{A}} \cdot A_{\mathrm{f}}}
$$

where $A_{\mathrm{f}}$ and $K_{\mathrm{A}}$ respectively represent the molar concentration of the polyphenol unbound and the binding constant of the polyphenol on the site of HSA, on the assumption of the competitive relationship between the drug and the polyphenol. Thus, $K_{\mathrm{A}}$ was estimated based on the equation

$$
K_{\mathrm{A}}=\left(\frac{K}{K^{\prime}}-1\right) / A_{\mathrm{f}}
$$

\section{Results}

Protocatechuic Acid and Its Methyl Derivatives The induced Cotton effect for PB-HSA according to the binding of PB to HSA was observed as shown in Fig. 2. Magnitudes of the induced Cotton effects were dependent on the concentration of $\mathrm{PB}$, when the monitoring wavelength was set at

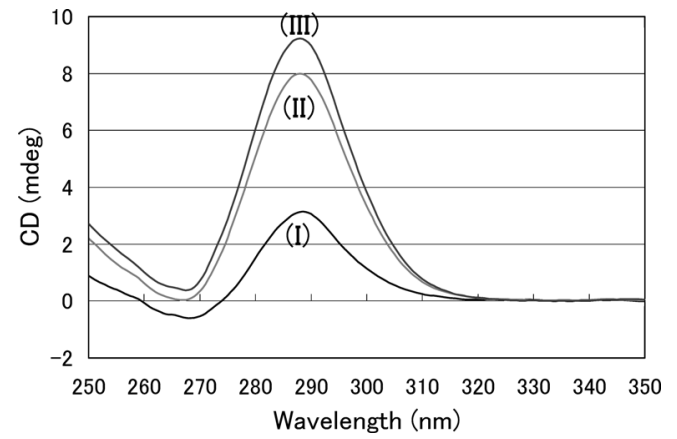

Fig. 2. Induced CD for PB-HSA

The presence of (I) $6.49 \times 10^{-5} \mathrm{M}$, (II) $1.95 \times 10^{-4} \mathrm{M}$, and (III) $3.24 \times 10^{-4} \mathrm{M}$ of PB in the solution of HSA $\left(6.02 \times 10^{-5} \mathrm{M}\right)$ caused the induced $\mathrm{CD}$, which was obtained by subtracting the CD for HSA from those of the combinations of HSA and PB.

\section{$290 \mathrm{~nm}$ (Fig. 3A)}

This induced Cotton effect was suppressed by the addition of protocatechuic acid, as shown in Fig. 3A, and a double-reciprocal plot analysis of the effect in the absence and presence of protocatechuic acid showed that the suppression of the binding of PB-HSA due to protocatechuic acid was competitive (Fig. 3B), indicating that protocatechuic acid binds to site I.

In an analogous manner, the effect of adding protocatechuic acid on the induced CD due to the combination DPHSA was examined via monitoring at $320 \mathrm{~nm}$. The induced CD was suppressed by the addition of protocatechuic acid (Fig. 3C), and the double-reciprocal plot analysis indicated that the interaction of DP and protocatechuic acid on binding to HSA was competitive (Fig. 3D). The binding of protocatechuic acid to site II was thus demonstrated.

The addition of DG to the induced Cotton effect due to protocatechuic acid $\left(0-5.19 \times 10^{-4} \mathrm{M}\right)$ and HSA was monitored at $260 \mathrm{~nm}$, and any observable effect was revealed by the addition of DG $\left(1.96 \times 10^{-4} \mathrm{M}\right)$ (data not shown).

Then, we examined the effects of vanillic acid and isovanillic acid on the PB-HSA and DP-HSA interactions, since methylation of phenolic compounds is assumable on the metabolic process.

The results for the addition of vanillic acid to PB-HSA and DP-HSA solutions at each concentration of PB and DP were analyzed using double-reciprocal plots as shown in Figs. 4A and $\mathrm{B}$. These data indicated that vanillic acid binds to site I and site II on HSA, competitively. In an analogous way, the effects of adding isovanillic acid to PB-HSA and DP-HSA solutions are shown in Figs. $4 \mathrm{C}$ and D as their double-reciprocal plots. Competitive binding of isovanillic acid to site I and site II on HSA was thus demonstrated. The induced Cotton effects for the combinations of vanillic acid-HSA and isovanillic acid-HSA were found to be similar to that observed for the combination protocatechuic acid-HSA, and the addition of DG did not affect these interactions (data not shown).

The changes in the binding constants for PB-HSA and for DP-HSA were also shown in Figs. 3 and 4. The decreasing effects of the addition of the polyphenolic compounds on the binding constant $K$ for PB-HSA showed large differences depending on their structures as seen in Table 1. This result indicated that the binding of these polyphenolic metabolites to site I on HSA was affected by methylation and the location 
A

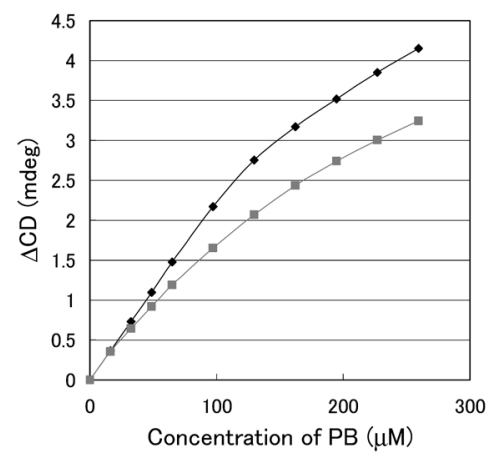

$\mathrm{C}$

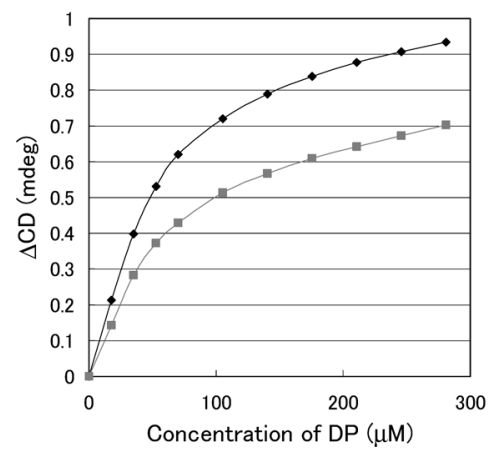

B

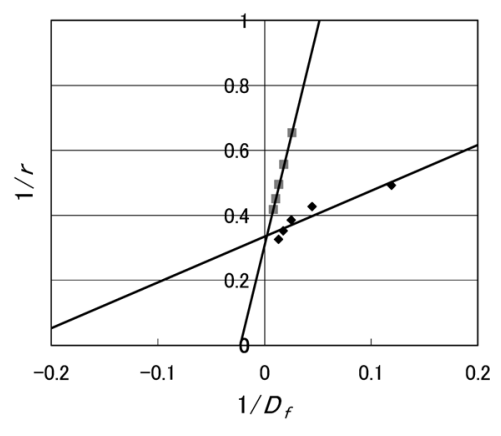

$\mathrm{D}$

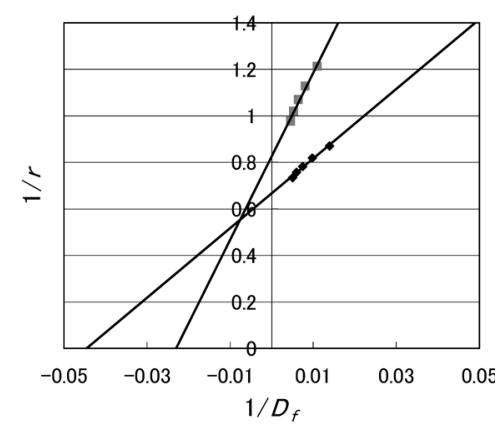

Fig. 3. Effects of Adding Protocatechuic Acid on the PB-HSA-Induced CD and on the DP-HSA-Induced CD

(A) Relationship between the concentration of PB and the amplitudes of the induced CD at $290 \mathrm{~nm}$ for the combination of HSA $\left(6.02 \times 10^{-5} \mathrm{M}\right)$ and PB in the absence $(\bullet)$ or presence $\left(\square, 9.73 \times 10^{-4} \mathrm{M}\right)$ of protocatechuic acid. (B) Double-reciprocal plots based on the induced CD. Plots in the absence $(\$)$ or presence of $9.73 \times 10^{-4} \mathrm{M}(\square)$ of protocatechuic acid on the binding of PB-HSA are shown. The binding constant $(K)$ in the absence and that in the presence of protocatechuic acid were $2.4 \times 10^{5} \mathrm{M}^{-1}$ and $2.3 \times 10^{4} \mathrm{M}^{-1}$, respectively. (C) Relationship between the concentration of DP and the amplitudes of the induced CD at $320 \mathrm{~nm}$ for the combination of HSA $\left(6.02 \times 10^{-5} \mathrm{M}\right)$ and DP in the absence $(\$)$ or presence $\left(\square, 9.73 \times 10^{-4} \mathrm{M}\right)$ of protocatechuic acid. (D) Double-reciprocal plots based on the induced CD. Plots in the absence $(\$)$ or presence of $9.73 \times 10^{-4} \mathrm{M}(\square)$ of protocatechuic acid on the binding of DP-HSA are shown. The $K$ values in the absence and in the presence of protocatechuic acid were $4.4 \times 10^{4} \mathrm{M}^{-1}$ and $2.3 \times 10^{4} \mathrm{M}^{-1}$, respectively.

of the methylation. Increase of the lipophilicity accompanied by the methylation may strengthen the affinity of the compound to this site. On the other hand, the effects of these three compounds on the binding constant for DP-HSA were almost the same.

Gallic Acid and 4-O-Methylgallic Acid The induced Cotton effects due to the interactions of PB-HSA and DPHSA were not affected by the addition of gallic acid at the concentration examined. However, the addition of 4-Omethylgallic acid to the PB-HSA solutions caused a decrease of the induced Cotton effect as shown in Fig. 5A. The double-reciprocal plot analysis in Fig. 5B showed that the effect was competitive, indicating that $4-O$-methylgallic acid binds to site I on HSA. In this case, the hydroxylation of isovanillic acid weakened the decreasing effect on the binding constant $K$ for PB-HSA (Table 1 ).

Its addition to DP-HSA solutions, however, did not affect the interaction. The induced Cotton effects for the combination 4-O-methylgallic acid and HSA was not affected by the addition of DG, which is a site III-binding drug (data not shown).

Ellagic Acid and Urolithin A Ellagic acid, which is formed from ellagitannins, is also a constituent of various food and medicinal plants. ${ }^{15)}$ During metabolic processing of hydrolyzable tannins, ellagic acid is also assumed to be produced from gallic acid under certain oxidative conditions.
Table 1. Binding Constants for PB-HSA and DP-HSA in the Absence $(K)$ and Presence $\left(K^{\prime}\right)$ of the Polyphenolic Metabolites

\begin{tabular}{ccccc}
\hline \hline $\begin{array}{l}\text { Site-specific binding drugs } \\
\text { + Polyphenolic metabolites }\end{array}$ & $K$ & $K^{\prime}$ & $K / K^{\prime}$ & $K_{\mathrm{A}}{ }^{a)}$ \\
& & & & \\
\hline Phenylbutazone (PB) & $2.4 \times 10^{5}$ & & & \\
$\quad$ + Protocatechuic acid & & $2.3 \times 10^{4}$ & $1.0 \times 10$ & $9.7 \times 10^{3}$ \\
$\quad$ + Vanillic acid & & $8.8 \times 10^{3}$ & $2.7 \times 10$ & $2.9 \times 10^{4}$ \\
+ Isovanillic acid & & $4.0 \times 10^{3}$ & $6.0 \times 10$ & $6.6 \times 10^{4}$ \\
$\quad$ + 4-O-Methylgallic acid & & $3.2 \times 10^{4}$ & 7.5 & $8.0 \times 10^{3}$ \\
Diazepam (DP) & $4.4 \times 10^{4}$ & & & \\
+ Protocatechuic acid & & $2.3 \times 10^{4}$ & 1.9 & $9.4 \times 10^{2}$ \\
+ Vanillic acid & & $2.3 \times 10^{4}$ & 1.9 & $1.0 \times 10^{3}$ \\
+ Isovanillic acid & & $2.6 \times 10^{4}$ & 1.7 & $7.7 \times 10^{2}$ \\
\hline
\end{tabular}

a) $K_{\mathrm{A}}$ for each of the polyphenolic metabolites on the binding to the corresponding site of HSA, which was estimated from $K$ and $K^{\prime}$. See text.

Urolithin A is reportedly a metabolite of ellagic acid and is assumed to be produced by the actions of intestinal bacteria. ${ }^{16)}$ The present study revealed that the effects of adding these two compounds to the solutions of PB-HSA and DPHSA were negligible on the induced Cotton effects (data not shown). Therefore, ellagic acid and urolithin A do not bind specifically on site I or site II on HSA.

\section{Discussion}

The CD spectral method has been shown to be an effective 
A

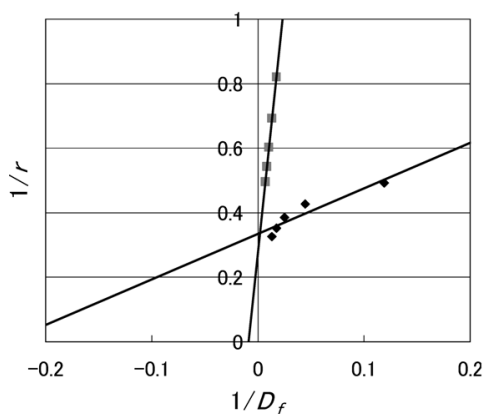

$\mathrm{C}$

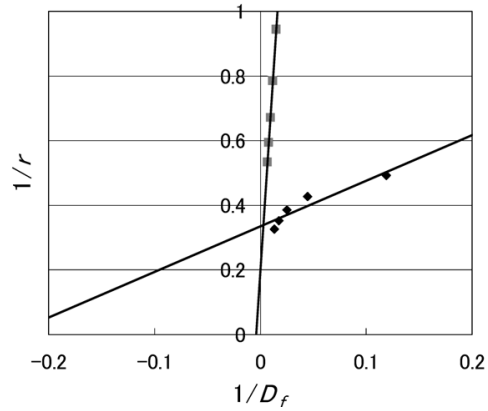

B

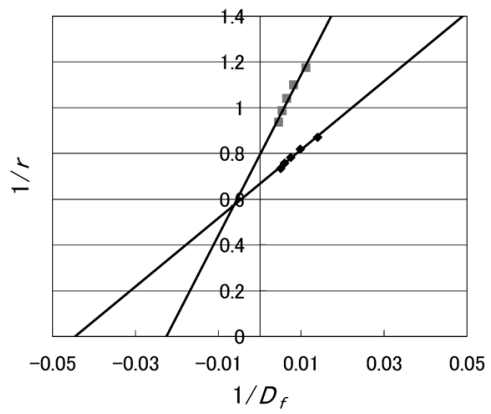

$\mathrm{D}$

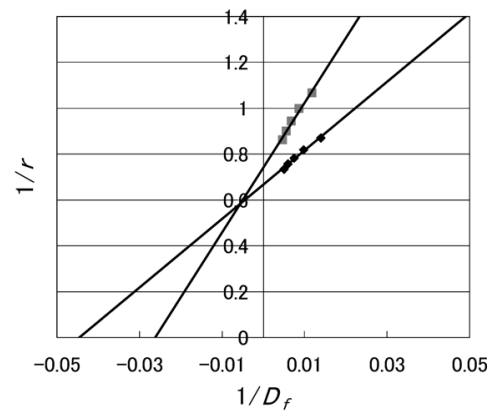

Fig. 4. Competitive Relationship of Vanillic Acid with PB (A) and with DP (B) and That of Isovanillic Acid with PB (C) and with DP (D) on HSA

(A) Double-reciprocal plots based on the induced CD of PB-HSA at $290 \mathrm{~nm}$ in the absence $(\bullet)$ or presence of $8.92 \times 10^{-4} \mathrm{M}(\square)$ of vanillic acid are shown. The $K$ value for the PB-HSA binding in the presence of vanillic acid was $8.8 \times 10^{3} \mathrm{M}^{-1}$. (B) The plots of DP-HSA at $320 \mathrm{~nm}$ in the absence $(\checkmark)$ or presence of $8.92 \times 10^{-4} \mathrm{M}(\square)$ of vanillic acid are shown. The $K$ value for the DP-HSA binding in the presence of vanillic acid was $2.3 \times 10^{4} \mathrm{M}^{-1}$. (C) The plots of PB-HSA at $290 \mathrm{~nm}$ in the absence $(\bullet)$ or presence of $8.92 \times 10^{-4} \mathrm{M}$ ( $\square$ ) of isovanillic acid are shown. The $K$ value for the PB-HSA binding in the presence of isovanillic acid was $4.0 \times 10^{3} \mathrm{M}^{-1}$. (D) The plots of DP-HSA at $320 \mathrm{~nm}$ in the absence ( $)$ or presence of $8.92 \times 10^{-4} \mathrm{M}(\square)$ of isovanillic acid are shown. The $K$ value for the DP-HSA binding in the presence of isovanillic acid was $2.6 \times 10^{4} \mathrm{M}^{-1}$.

A

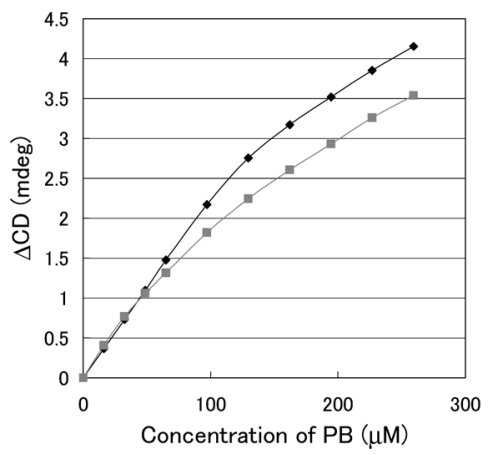

B

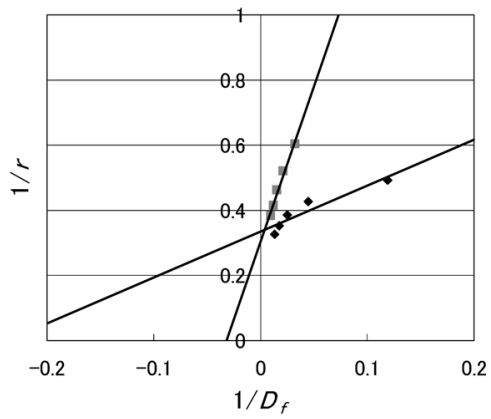

Fig. 5. Effects of Adding 4-O-Methylgallic Acid on the PB-HSA-Induced CD

(A) Relationship between the concentration of PB and the amplitudes of the induced CD at $290 \mathrm{~nm}$ for the combination of $\mathrm{HSA}\left(6.02 \times 10^{-5} \mathrm{M}\right)$ and $\mathrm{PB}$ in the absence $(\bullet)$ or presence $\left(\square, 8.15 \times 10^{-4} \mathrm{M}\right)$ of $4-O$-methylgallic acid. (B) Double-reciprocal plots based on the induced CD. Plots in the absence $(\checkmark)$ or presence $\left(\square, 8.15 \times 10^{-4} \mathrm{M}\right)$ of $4-O$-methylgallic acid are shown. The $K$ value for the PB-HSA binding in the presence of $4-O$-methylgallic acid was $3.2 \times 10^{4} \mathrm{M}^{-1}$.

technique in clarifying the binding of polyphenolic compounds to HSA using only a small amount of sample.

The present investigation revealed that protocatechuic acid and its partially methylated products bind to sites I and II on HSA. In contrast, the addition of a hydroxyl group from protocatechuic acid yielding gallic acid did not result in competitive binding to site I or site II. Since the binding of gallic acid to HSA was observed using gel electrophoretic analysis, ${ }^{17)}$ binding occurred on the surface of HSA, but not to these specific sites. In addition, analogous assumptions were also possible for the binding of ellagic acid and urolithin A.

4-O-Methylgallic acid, regarded as a compound resulting from adding a hydroxyl group to isovanillic acid, caused the disappearance of the characteristic binding site II on HSA and showed specific binding to site I on HSA. This compound is also regarded as a product of the partial methylation of gallic acid, which did not show specific binding to HSA as described above. Such a change in the binding to HSA suggested the importance of methylation by COMT in the metabolic process.

In conclusion, the competitive/noncompetitive relationships of the metabolites from polyphenolic constituents in plants differ from each other depending on their structures. 


\section{References}

1) Stangl V., Lorenz M., Stangl K., Mol. Nutr. Food Res., 50, 218-228 (2006).

2) Apak R., Güçlü K., Ozyürek M., Karademir S. E., J. Agric. Food Chem., 52, 7970_7981 (2004).

3) Cao D., Zhang Y., Zhang H., Zhong L., Qian X., Rapid. Commun. Mass Spectrom., 23, 1147-1157 (2009).

4) Lin L. C., Wang M. N., Tsai T. H., Chem. Biol. Interact., 174, 177182 (2008).

5) Nozaki A., Hori M., Kimura T., Ito H., Hatano T., Chem. Pharm. Bull., 57, 224-228 (2009).

6) Gonthier M. P., Donovan J. L., Texier O., Felgines C., Remesy C., Scalbert A., Free Radic. Biol. Med., 35, 837-844 (2003).

7) Nakamura Y., Tsuji S., Tonogai Y., J. Agric. Food Chem., 51, 331339 (2003).

8) Booth A. N., Masri M. S., Robbins D. J., Emerson O. H., Jones F. T., DeEds F., J. Biol. Chem., 234, 3014-3016 (1959).
9) Cerdá B., Espín J. C., Parra S., Martínez P., Tomás-Barberán F. A., Eur. J. Nutr., 43, 205-220 (2004).

10) Seeram N. P., Henning S. M., Zhang Y., Suchard M., Li Z., Heber D., J. Nutr., 136, 2481-2485 (2006).

11) Ito H., Iguchi A., Hatano T., J. Agric. Food Chem., 56, 393-400 (2008).

12) John P. D., Can. J. Chem., 53, 343-349 (1975)

13) Rosen A., Biochem. Pharmacol., 19, 2075-2081 (1970).

14) Motoya T., Thevanayagam L. N., Blaschke T. F., Au S., Stone J. A., Jayewardene A. L., Chi J., Aweeka F. T., HIV Medicine, 7, 122-128 (2006).

15) Määttä-Riihinen K. R., Kamal-Eldin A., Törrönen A. R., J. Agric. Food Chem., 52, 6178-6187 (2004).

16) Cerdá B., Periago P., Espín J. C., Tomás-Barberán F. A., J. Agric. Food Chem., 53, 5571-5576 (2005).

17) Kusuda M., Hatano T., Yoshida T., Biosci. Biotech. Biochem., 70, $152-160$ (2006). 